\title{
Arginine ADP-ribosyltransferase 1 promotes angiogenesis in colorectal cancer via the PI3K/Akt pathway
}

\author{
LIAN YANG, MING XIAO, XIAN LI, YI TANG and YA-LAN WANG \\ Department of Pathology, Molecular Medicine and Cancer Research Center, \\ Chongqing Medical University, Chongqing 400016, P.R. China
}

Received June 29, 2015; Accepted January 18, 2016

DOI: $10.3892 /$ ijmm.2016.2473

\begin{abstract}
Arginine adenosine diphosphate (ADP)-ribosyltransferase 1 (ART1) is known to play an important role in many physiological and pathological processes. Previous studies have demonstrated that ART1 promotes proliferation, invasion and metastasis in colon carcinoma. However, it was unclear whether ART1 is involved in angiogenesis in cases of colorectal cancer (CRC). In the present study, lentiviral vector-mediated ART1-cDNA or ART1-shRNA were transfected into LoVo cells, and the LoVo cells transfected with ART1-cDNA or ART1-shRNA were co-cultured with human umbilical vein endothelial cells (HUVECs) to determine the influence of ART1 on HUVECs. The proliferation, migration and angiogenesis of HUVECs were monitored using a cell counting kit- 8 assay, a Transwell migration assay and immunohistochemical analysis in intrasplenic allograft tumors, respectively. Hypoxia-inducible factor $1-\alpha$ (HIF-1 $\alpha)$, total (t-) Akt, phosphorylated (p-)Akt, vascular endothelial growth factor (VEGF) and basic fibroblast growth factor (bFGF) expression levels were detected via western blot analysis. Our results revealed that HUVECs which were co-cultured with ART1-cDNA LoVo cells showed higher proliferation, migration and angiogenic abilities, but a reduction was noted in those cultured with ART1-shRNA LoVo cells; p-Akt, HIF-1 $\alpha$, VEGF and bFGF expression was increased in HUVECs cultured with ART1-cDNA-transfected LoVo cells, but reduced in ART1shRNA-transfected LoVo cells. In a mouse xenograft model, we noted that the tumor microvessel density (MVD) was significantly increased in intrasplenic transplanted ART1-cDNA CT26 tumors but decreased in intrasplenic transplanted ART1-shRNA tumors. These data suggest that ART1 promoted the expression of HIF-1 $\alpha$ via the Akt pathway in tumor cells. It
\end{abstract}

Correspondence to: Dr Ya-Lan Wang, Department of Pathology, Molecular Medicine and Cancer Research Center, Chongqing Medical University, Chongqing 400016, P.R. China

E-mail: wangyalan074@126.com

Key words: colorectal cancer, LoVo cells, human umbilical vein endothelial cells, arginine adenosine diphosphate-ribosyltransferase 1, angiogenesis also upregulated VEGF and bFGF and enhanced angiogenesis in HUVECs. Thus, we suggest that ART1 plays an important role in the invasion of CRC cells and the metastasis of CRC.

\section{Introduction}

Mono-adenosine diphosphate (ADP)-ribosyltransferases (ARTs) have been noted to catalyze ADP-ribosylation, which transfers an ADP-ribose from nicotinamide adenine dinucleotide $\left(\mathrm{NAD}^{+}\right)$to various amino acid residues, thus altering protein function and playing an important role in physiological and pathological processes such as signal transduction, cellular differentiation and proliferation, protein secretion and transport, cellular necrosis and apoptosis (1). Seven monoADP-ribosyltransferases (ART1-ART7) have previously been found in mammalians, and five members (ART1, ART2, ART5, ART6 and ART7) are specific to arginine $(2,3)$. ART1 was first discovered in bacteria, viruses and eukaryotic cells (4), and it was subsequently found in human skeletal muscles, cardiac muscle cells, white blood cells and airway epithelial cells (5-7). The gene which codes for ART1 is located on human chromosome 11P15, and its target proteins include integrin $\alpha 7, \alpha \mathrm{L}$ (CD11a), $\beta 2$ (CD18), human neutrophil peptide-1 (HNP-1), basic fibroblast growth factor (bFGF) and platelet-derived growth factor (PDGF)-BB (8-12).

In non-neoplastic diseases, ARTs are related to the occurrence of acute inflammation of the intestinal mucosa (13). When mono-ADP-ribosylation is inhibited, the release of inflammatory mediators by monocytes and macrophages is inhibited, suggesting that ARTs promote inflammatory development (14). Furthermore, ART1 specifically alters HNP-1 activity, which reduces antibacterial and antitoxic ability of HNP-1 (15). When T lymphocytes are transfected with ART1 in the presence of $\mathrm{NAD}^{+}$, the function of the $\mathrm{T}$ cell receptor is altered by the lymphocyte function-associated factor, thus affecting lymphocyte function and suggesting that ART1 acts in an extensive capacity $(9,16)$. Furthermore, gastric cancer researchers have found that Helicobacter pylori modulates ART activity, leading to modifications in membrane conformation and possibly promoting the development of gastric cancer (17). In the lung cancer cell line A549, ART1 is highly expressed; ART1 and ART4 are upregulated upon human Toll-like receptor (hTLR) stimulation, thus suggesting that ARTs are important in the development of lung cancer (18). 
Previously we demonstrated that ART1 was highly expressed in human colorectal cancer (CRC), and that its expression was positively correlated with the expression of angiogenic factors such as integrin $\alpha \mathrm{V} \beta 3$, vascular endothelial growth factor (VEGF) and bFGF (19). It has previously been suggested that ART1 has the ability to promote angiogenesis in CRC. Several previous studies have reported that the phosphoinositide 3-kinase (PI3K)/Akt pathway not only promotes tumor cell proliferation and inhibits apoptosis, but is also closely related to tumor angiogenesis. This mechanism may be involved in the activation of the PI3K/Akt pathway, which subsequently upregulates the expression of hypoxia-inducible factor $1-\alpha$ (HIF-1 $\alpha$ ) and promotes transcription and secretion of VEGF, and eventually leads to promote tumor angiogenesis through VEGF binding to vascular endothelial cell surface receptors $(20,21)$. It is unclear whether ART1 is involved in VEGF, bFGF and HIF-1 $\alpha$ expression, which is regulated by the PI3K/Akt pathway, in CRC cells thus far.

In the present study, LoVo cells transfected with ART1-cDNA or with ART1-shRNA were co-cultured with human umbilical vein endothelial cells (HUVECs), and subsequently HUVEC proliferation, migration and vascular-forming abilities were all examined. Additionally, the effect of ART1 on tumor microvessel density (MVD) was examined in vivo. The expression levels of total (t-)Akt, phosphorylated (p-)Akt, HIF-1 $\alpha$, VEGF and bFGF were further detected in order to characterize the molecular mechanisms of ART1 during tumor angiogenesis.

\section{Materials and methods}

Cell and lentiviral transfection. The LoVo cell line, a human colon cancer cell line, and HUVECs were kindly provided by Professor Wei-Xue Tang, Chongqing Medical University (Chongqing, China). The mouse colon cancer cell line CT26 was kindly provided by Professor Yu-Quan Wei, Sichuan University (Chengdu, China). ART1 was cloned into a pGCSIL-GFP lentiviral vector in order in order to generate both pGCSIL-GFP-vshART1 and pGCSIL-GFP-ART1cDNA. The ART1-shRNA interference sequence was GCCAACAAAGTATACGCGGAT. The primers of ART1-cDNA used were as follows: primer 1, GAGGAT CCCCGGGTACCGGTCGCCACCATGAAGATTCCTGCTA TGATG; primer 2, TCACCATGGTGGCGACCGGACA TCGGGTAAGTTGCTG. The pGCL-GFP-vshART1 lentiviral vector, ART1-cDNA lentiviral vector and empty lentiviral vector were purchased from GeneChem, Inc. (Shanghai, China). The human CRC cell line LoVo was cultured and transfected according to the manufacturer's instructions (GeneChem, Inc.). LoVo cells were seeded in 24-well plates at a density of $3 \times 10^{4}$ cells/well, transfected with $10 \mu \mathrm{l}$ lentiviral vector and observed under a fluorescence microscope $96 \mathrm{~h}$ post-transfection until the infection rate reached $80 \%$. Transfection efficiency was detected under a fluorescence microscope (Leica DMI4000B; Leica Microsystems GmbH, Wetzlar, Germany) after $96 \mathrm{~h}$. The efficiency of the ART1-cDNA and ART1-shRNA lentivirus transfected into LoVo cells was detected by reverse transcription (RT)PCR and western blot analysis. The murine CRC cell line CT26 was cultured and transfected as previously described by
Tang et al (22). The group which were transfected with ART1-shRNA lentiviral and the group transfected with ART1-cDNA lentiviral vector were set as experimental groups. The empty lentiviral vector transfected group (LV-control group) and untransfected group were set as control groups.

$R T-P C R$. Total RNA was separately extracted from untransfected LoVo cells, LV-control LoVo cells, ART1-cDNA- and ART1-shRNA-transfected LoVo cells with TRIzol reagent (Takara, Dalian, China) and reverse transcribed into DNA. ART1 gene expression was detected using oligonucleotide primers which were designed and produced by Sangon Biotech Co. (Shanghai, China): ART1, 5'-ACCTTCTTCGGTA TCTGGACCT-3' (F1) and 5'-TAAGTTGCTGGAGACCTG GATT-3' (R1); and $\beta$-actin acted as the internal control gene, 5'-ATATCGCTGCGCTGGTC GTC-3' (F1) and 5'-AGGATGG CGTGAGGGAGAGC-3' (R1). Using a one-step RT-PCR kit (Takara), in total, 30 PCR cycles were used for the amplification of RT products $\left(94^{\circ} \mathrm{C}\right.$ for $30 \mathrm{sec}, 60^{\circ} \mathrm{C}$ for $30 \mathrm{sec}$ and $72^{\circ} \mathrm{C}$ for $25 \mathrm{sec}$ ). Finally, the amplifiation products were electrophoresed on $2 \%$ agarose gels (Genview, Tallahasse, FL, USA). The above experiment was performed in triplicate

Analysis of HUVEC proliferation. In the present study,HUVEC proliferation was detected as previously described (23). The single-cell suspensions of HUVECs were cultured in a 96-well culture plate at a final concentration of $2 \times 10^{3}$ cells/well in Dulbecco's modified Eagle's medium with $20 \%$ fetal bovine serum (both purchased from Gibco-BRL, Gaithersburg, MD, USA) for $24 \mathrm{~h}$. HUVECs were subsequently co-cultured with supernatant fluid of LoVo cells in ratios of $0,20,40,60,80$ and $100 \% 48 \mathrm{~h}$ later. This was followed by the addition of $10 \mu \mathrm{l}$ of Cell Counting kit (CCK-8; KeyGen Biotechnology, Nanjing, China) reagent to each well, incubation for $4 \mathrm{~h}$ and detection at an OD of $450 \mathrm{~nm}$ by universal microplate reader (Bio-Tek Instruments Inc., Winooski, VT, USA). The assays were repeated in triplicate.

HUVECs migration analysis. HUVEC migration was detected using 8.0-mm pore size Transwell inserts (Costar, Milpitas, CA, USA) as previously described (24). The Transwell semipermeable membrane was coated with diluted Matrigel (Sigma, Ronkonkoma, NY, USA) incubated at $37^{\circ} \mathrm{C}$ for $30 \mathrm{~min}$ to solidify. LoVo single-cell suspension was seeded into the lower chamber at a concentration of $1 \times 10^{5}$ cells/well and incubated overnight in RPMI-1640 with $10 \%$ FBS (both from Gibco-BRL, Gaithersburg, MD, USA). HUVEC single-cell suspension was seeded in the upper chamber at a concentration of $1 \times 10^{5}$ cells/well and incubated under conventional culture conditions. At 6 and $12 \mathrm{~h}$ later, the cells on the upper surface of the membrane were carefully scraped off and the migrant cells which adhered to the lower surface were fixed with $4 \%$ methanol for $15 \mathrm{~min}$ at room temperature, and stained with $0.1 \%$ crystal violet for $3 \mathrm{~min}$. The number of cells on the lower surface of the membrane was counted in five different fields using an inverted microscope at a magnification of $x 200$. The experiment was repeated in triplicate.

Analysis of HUVEC lumen-forming ability. A 3D collagen cell culture system (Millipore, Inc., Billerica, MA,USA) was utilized; 
we proportionally combined collagen solution, $5 \mathrm{X}$ medium and neutralization solution, with $150 \mu \mathrm{l}$ of the mixture added to each well of a 24-well plate. The plate was then incubated at $37^{\circ} \mathrm{C}$ for $60 \mathrm{~min}$ in order to solidify. The HUVEC single cell suspension was then seeded into each well at a concentration of $5 \times 10^{5}$ cells/ well and mixed with different ratios $(0,10,20$ and 50\%) of supernatant from transfected LoVo cells, cultured for $72 \mathrm{~h}$ and observed under the microscope, with the total length randomly selected for 5 lumen, and the total length was measured (25). The experiment was repeated in triplicate.

Transplanted tumor mouse model. Sixteen BALB/c female mice (Chongqing Medical University Laboratory Animal Center, Chongqing, China), 6-8 weeks old and weighing 18-22 g, were randomly divided into four groups: the group transfected with ART1-shRNA lentiviral vector, the group transfected with ART1-cDNA lentiviral vector, a group transfected with an empty lentiviral and the unransfected group. Mice were inoculated as previously described (26). Following anesthesia an intraperitoneal injection of $2 \%$ chloral hydrate (Beijing DingGuo Biological Technology Co., Ltd., Beijing, China), the mice were fixed in a supine position, and an incision was made into the abdominal cavity from the left upper quadrant axillary midline to expose the spleen. The mice were administered the CT26 cells $\left(5 \times 10^{6}\right)$ in single suspension/ mouse via a subcapsular injection into the spleen. Fourteen days later, the mice were sacrificed by cervical dislocation and the metastatic spleen tissues were removed and fixed with $4 \%$ paraformaldehyde, and paraffin-embedded tissues were cut into slices for H\&E staining and immunohistochemistry.

All animal experiments were performed after obtaining approval from the Ethics Committee of Chongqing Medical University. Appropriate care was taken to minimize animal suffering. The disposition of the animals at the end of the study, euthanisa criteria and all experimental methods involving animals were in accordance with the code of practice for the care and use of animals for scientific purposes.

$H \& E$ staining and CD34 immunohistochemical assay. The tissue sections were dewaxed with xylene, and the slides were then soaked in graded concentrations of alcohol $(100,95,85$ and $70 \%$ ) for $5 \mathrm{~min}$, stained with hematoxylin for $3 \mathrm{~min}$ and eosin for $1 \mathrm{~min}$ (both from Beijing DingGuo Biological Technology Co., Ltd.) washing with water. Vascular endothelial cells were marked with CD34 (Wuhan Boster Biological Technology, Ltd., Wuhan, China) using an EnVision ${ }^{\mathrm{TM}}$ detection kit (DakoCytomation, Genetech, Beijing, China) following the manufacturer's protocols, as previously described (27). According to the method put forward by Weidner et al (27), areas with the most intense coloring were selected as 'hot spots', and the microvessels were counted under the microscope and imaged, with 3 'hot spots' counted per slice and averaged to obtain the slide MVD.

Western blot analysis. LoVo cells from each transfection group were collected; total protein was extracted according to protein extraction protocols (Beyotime P0013; Beyotime Biotechnology Co., Ltd. , Shanghai, China). Protein concentrations were determined using a BCA assay kit (Beyotime Biotechnology Co., Ltd. ) and equal amounts of total protein were separated via SDS-PAGE and transferred onto a PVDF membrane. The membrane was blocked at room temperature for $1 \mathrm{~h}$, and incubated with the primary antibody overnight at $4^{\circ} \mathrm{C}$. The primary antibodies, ART1 (AP2311a; Abgent Biotechnology, Inc. San Diego, CA, USA), HIF-1 $\alpha$ (20960-1-AP; Proteintech Group, Inc., Chicago, IL,

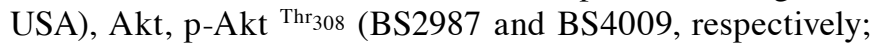
Bioworld Technology, Co., Ltd., St. Louis Park, MN, USA), VEGF, bFGF and $\beta$-actin (PB0084, BA0259 and BA2305, respectively; Wuhan Boster Biological Technology, Ltd.), were diluted to $1: 500,1: 1,000,1: 800,1: 600,1: 1,000,1: 1,000$ and 1:1,000, respectively. The membrane was washed with TBST and incubated with the secondary antibody (peroxidaseconjugated goat anti-rabbit $\mathrm{IgG}$, peroxidase-conjugated rabbit anti-goat IgG or peroxidase-conjugated goat anti-mouse IgG; ZDR-5306, ZDR-5308 and ZDR-5307, respectively; Beijing ZSGB Biotechnology Co., Ltd.) at a dilution of 1:1000 for $2 \mathrm{~h}$. The image was captured and imaged using a ChemiDoc XRS system (Bio-Rad, Hercules, CA, USA) using chemiluminescence (BeyoECL Plus; Beyotime Biotechnology Co., Ltd.) and analyzed using Quantity one software. The experiment was repeated in triplicate.

Statistical analysis. Statistical analysis was performed using SPSS 18.0 statistical software, and the data is expressed as the means $\pm \mathrm{SD}$; single factor compared analysis was used for analysis between groups, and the LSD method was used between two groups. A P-value $<0.05$ was considered to indicate a statistically significant difference.

\section{Results}

LoVo Cells transfected with lentivirus. LoVo cells were transfected with ART1-shRNA or ART1-cDNA lentiviral vector, and cells exhibited $80 \%$ fluorescence on the third and fifth days, respectively (Fig. 1A). ART1 expression in LoVo cells was detected by RT-PCR and western blot analysis. Compared with the untransfected and LV-control-transfected groups, ART1 mRNA and protein expression was significantly lower in the ART1-silenced groups and significantly higher in the ART1-cDNA-transfected group $(\mathrm{P}<0.05)$. However, there was no significant difference in ART1 mRNA and protein expression between un-transfected LoVo cells and LV-control-transfected LoVo cells $(\mathrm{P}>0.05)$ (Fig. 1B).

Effects of ART1-shRNA-transfected LoVo cells and ART1-cDNA-transfected LoVo cells on HUVEC proliferation. The proliferation capacity of HUVECs co-cultured with the supernatant fluid of ART1-shRNA LoVo cells was significantly decreased relative to the control group and untransfected group $(\mathrm{P}<0.05)$. By contrast, the proliferation capacity of HUVECs co-cultured with the supernatant fluid of ART1-cDNA LoVo cells was significantly higher $(\mathrm{P}<0.05)$, and the proliferation capacity increased with the increasing concentration of the LoVo supernatant. Furthermore, no significant difference between HUVECs co-cultured with the supernatant fluid of untransfected LoVo cells and LV-control LoVo cells was noted (P>0.05) (Fig. 2).

Effects of ART1-shRNA-transfected LoVo cells and ART1-cDNA-transfected LoVo cells on HUVEC migration. 

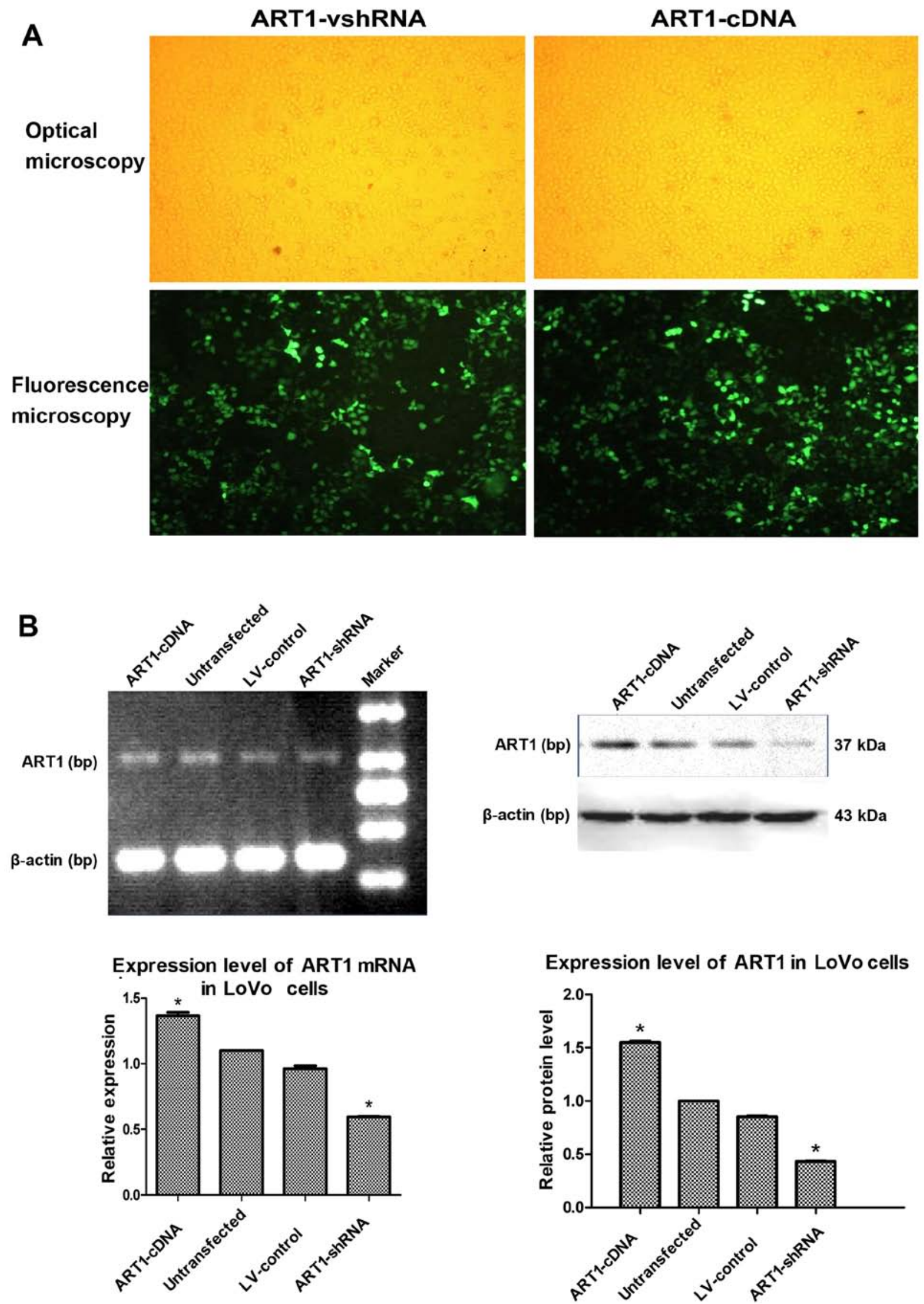

Figure 1. Lentiviral vector-mediated argenine-specific adenosine diphosphate (ADP)-ribosyltransferase 1 (ART1)-shRNA and ART1-cDNA are successfully transfected into LoVo cells. (A) LoVo cells transfected with lentiviral vector-mediated ART1-shRNA and ART1-cDNA. Ten microliters of lentiviral vector was transfected into LoVo cells, and the efficiency was observed under an optical microscope and fluorescence microscope after $96 \mathrm{~h}$, when the infection rate reached $80 \%$. (B) The expression levels of ART1 mRNA and protein in ART1-cDNA LoVo cells were compared with the ART1-shRNA LoVo cells, the lentivirus (LV)-control LoVo cells and the untransfected LoVo cells. ${ }^{*} \mathrm{P}<0.05$ vs. untransfected LoVo cells or LV-control LoVo cells; $\mathrm{n}=3$ experiments. 


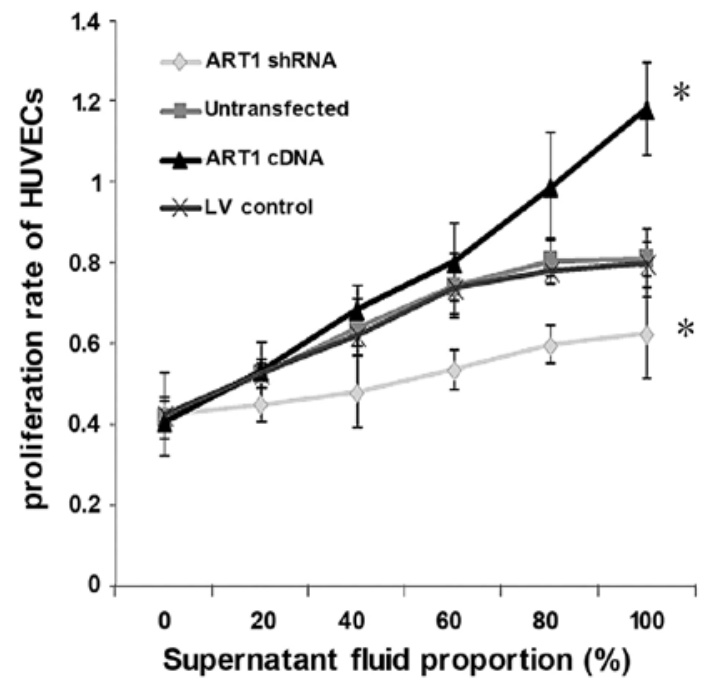

Figure 2. Proliferation ability of human umbilical vein endothelial cells (HUVECs) co-cultured with supernatant fluid of argenine-specific adenosine diphosphate (ADP)-ribosyltransferase 1 (ART1)-cDNA LoVo cells at ratios of $0,20,40,60,80$ and $100 \%$ was compared with those co-cultured with the supernatant fluid of ART1-shRNA LoVo cells, the lentivirus (LV)-control LoVo cells and the untransfected LoVo cells. ${ }^{*} \mathrm{P}<0.05$ vs. untransfected LoVo cells or LV-control LoVo cells; $\mathrm{n}=3$ experiments.
Compared with the control group, the migratory ability of HUVECs co-cultured with the ART1-shRNA-transfected cells was significantly decreased $(\mathrm{P}<0.05)$. By contrast, HUVEC migration increased after co-culture with ART1-cDAN LoVo cells $(\mathrm{P}<0.05)$. The difference between HUVECs co-cultured with untransfected LoVo cells and with LV-control LoVo cells was not significant $(\mathrm{P}>0.05)$. Moreover, after $12 \mathrm{~h}$, the majority of the HUVECs had passed through the Transwell membrane, and the difference was indistinguishable from the HUVECs co-cultured with the control LoVo cells ( $\mathrm{P}>0.05)$ (Fig. 3).

Effects of ART1-shRNA-transfected LoVo cells and ART1-cDNA-transfected LoVo cells on the blood vessel forming ability of HUVECs. HUVEC angiogenic abilities were significantly enhanced after incubation with the supernatant of ART1-cDNA-transfected LoVo cells. Both the number and length of vessels were significantly higher than those in the control group and untransfected group $(\mathrm{P}<0.05)$. Furthermore, the blood vessel lumens were mutually staggered. Moreover, HUVEC vascular forming ability was significantly reduced when co-cultured with the supernatant of ART1-silenced cells $(\mathrm{P}<0.05)$. There was no significant difference between

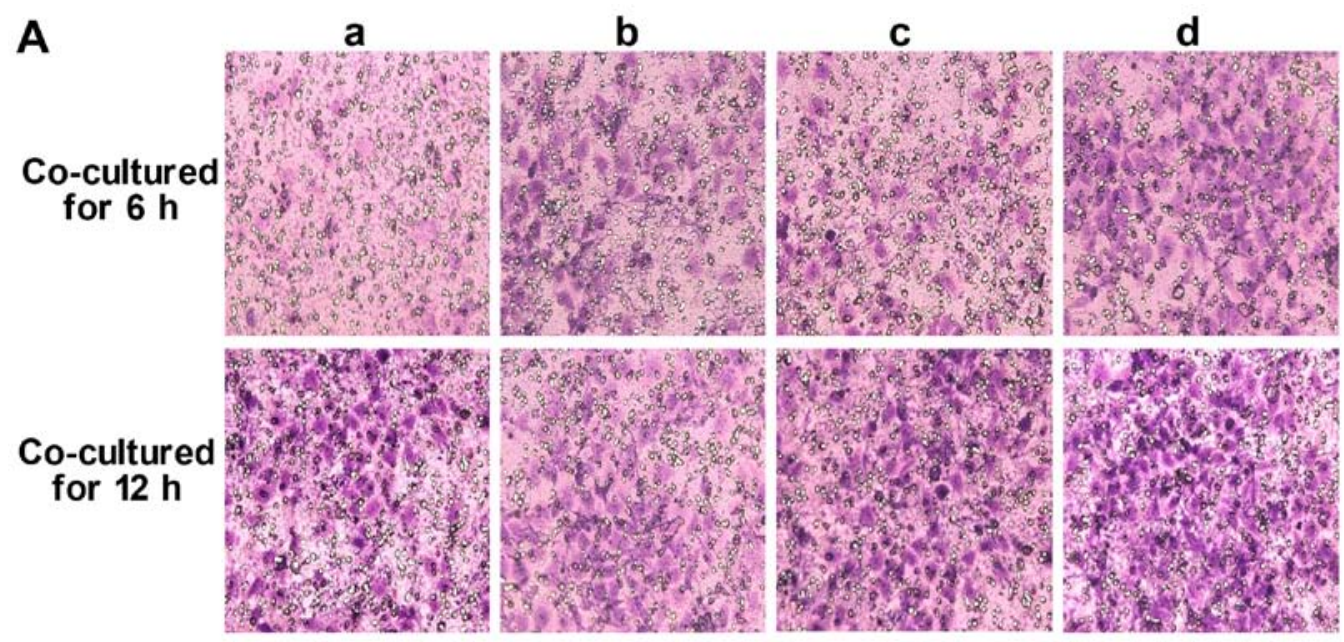

B

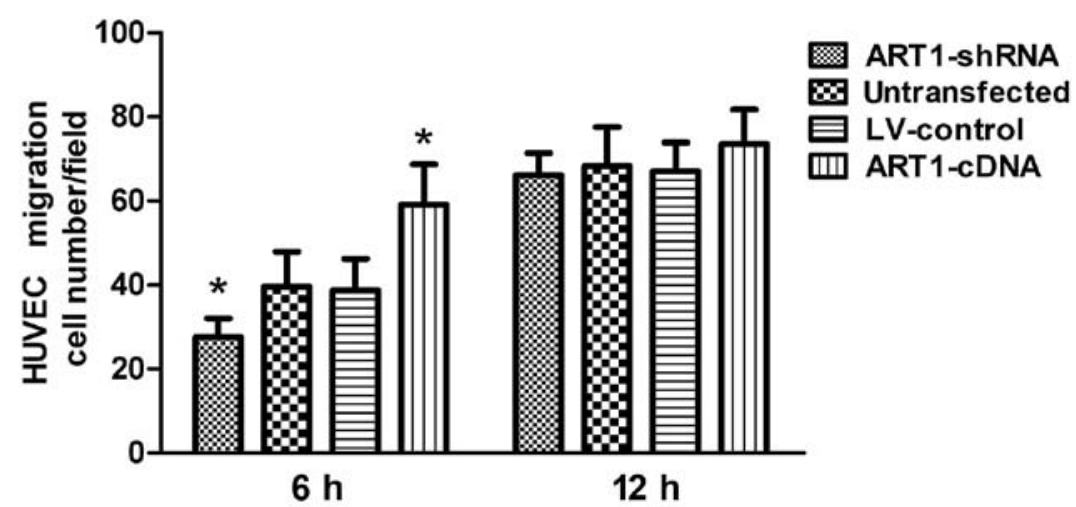

Figure 3. The effect of argenine-specific adenosine diphosphate (ADP)-ribosyltransferase 1 (ART1)-mediated LoVo cells on HUVEC migration. (A) The migratory ability of human umbilical vein endothelial cells (HUVECs) co-cultured with ART1-mediated LoVo cells. (a) HUVECs co-cultured with ART1shRNA LoVo cells; (b) HUVECs co-cultured with the untransfected LoVo cells; (c) HUVECs co-cultured with LV-control LoVo cells; (d) HUVECs co-cultured with the ART1-cDNA LoVo cells. (B) The comparison of migration of HUVECs co-cultured with ART1-mediated LoVo cells afer 6 and 12 h. ${ }^{*} \mathrm{P}<0.05$ vs. untransfected LoVo cells or lentivirus (LV)-control LoVo cells; $n=3$ experiments. 

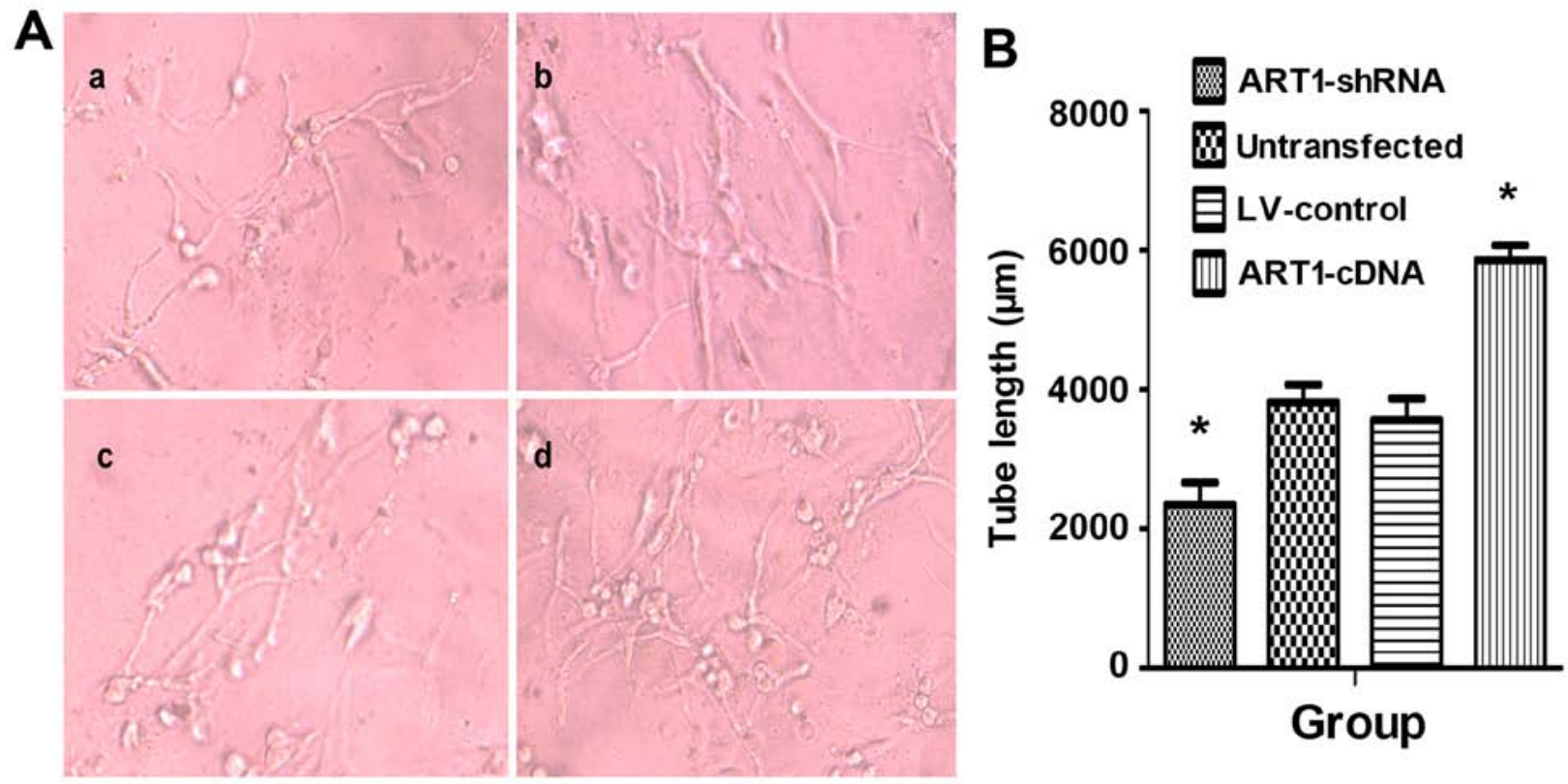

Figure 4. The effect of argenine-specific adenosine diphosphate (ADP)-ribosyltransferase 1 (ART1)-mediated LoVo cells on the blood vessel forming ability of human umbilical vein endothelial cells (HUVECs). (A) HUVEC angiogenic abilities when incubated with the supernatant of ART1-mediated LoVo cells. (a) HUVECs cultured with supernatant of ART1-shRNA LoVo cells; (b) HUVECs co-cultured with the untransfected LoVo cells; (c) HUVECs co-cultured with LV-control LoVo cells; (d) HUVECs co-cultured with the ART1-cDNA LoVo cells. (B) The tube length incubated with supernatant of ART1-cDNA LoVo cells was compared with incubated with the supernatant fluid of ART1-shRNA LoVo cells, the lentivirus (LV)-control LoVo cells and the untransfected LoVo cells. " $\mathrm{P}<0.05$ vs. untransfected or $\mathrm{LV}$-control group; $\mathrm{n}=3$ experiments.

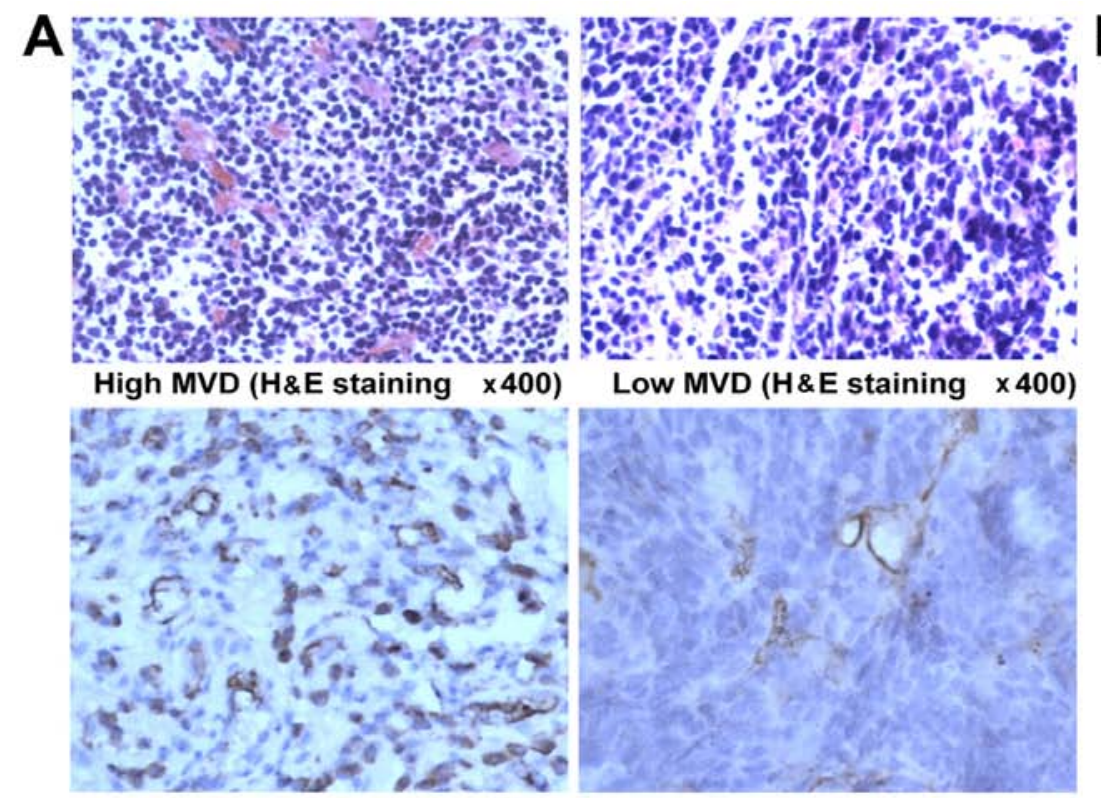

High MVD (CD34 IHC stain $\times 400)$ Low MVD (CD34 IHC stain $\times 400)$

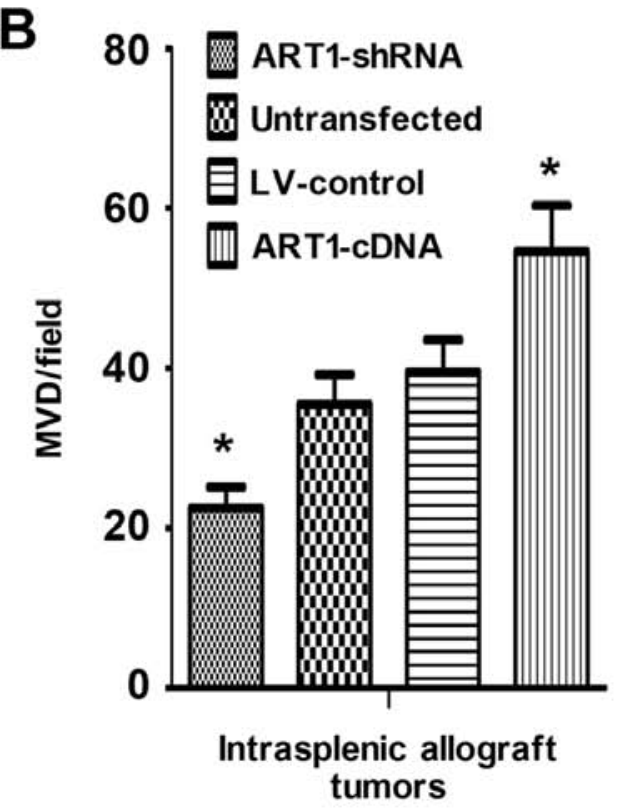

Figure 5. Microvessel density (MVD) in mice intrasplenic allograft tumor tissues. (A) The expression of CD34 indicated 'hot spots' and low MVD area in intrasplenic allograft tumors (H\&E and immunohistochemical staining; magnification x400). (B) The comparison of MVD in intrasplenic transplanted argenine-specific adenosine diphosphate (ADP)-ribosyltransferase 1 (ART1)-cDNA CT26 tumors with transplanted ART1-shRNA CT26 cells, LV-control CT26 cells and untransfected CT26 cells. " $\mathrm{P}<0.05$ vs. untransfected or LV-control CT26 cells; $\mathrm{n}=4$ mice in each group.

HUVECs incubated with the supernatant of untransfected LoVo cells and LV-control LoVo cells (P>0.05) (Fig. 4).

MVD in mice with transplanted tumor tissues. We noted that, compared with the control group, the MVD of tumors was significantly increased in mice which had undergone intras- plenic ART1-cDNA-transfection, whereas it was decreased in mice which had undergone intrasplenic transplantation with ART1-shRNA-transfected CT26 cells $(\mathrm{P}<0.05)$. However, the difference was not significant in the mice which were infected with untransfected CT26 cells and LV-control-transfected CT26 cells $(\mathrm{P}>0.05)$ (Fig. 5). 

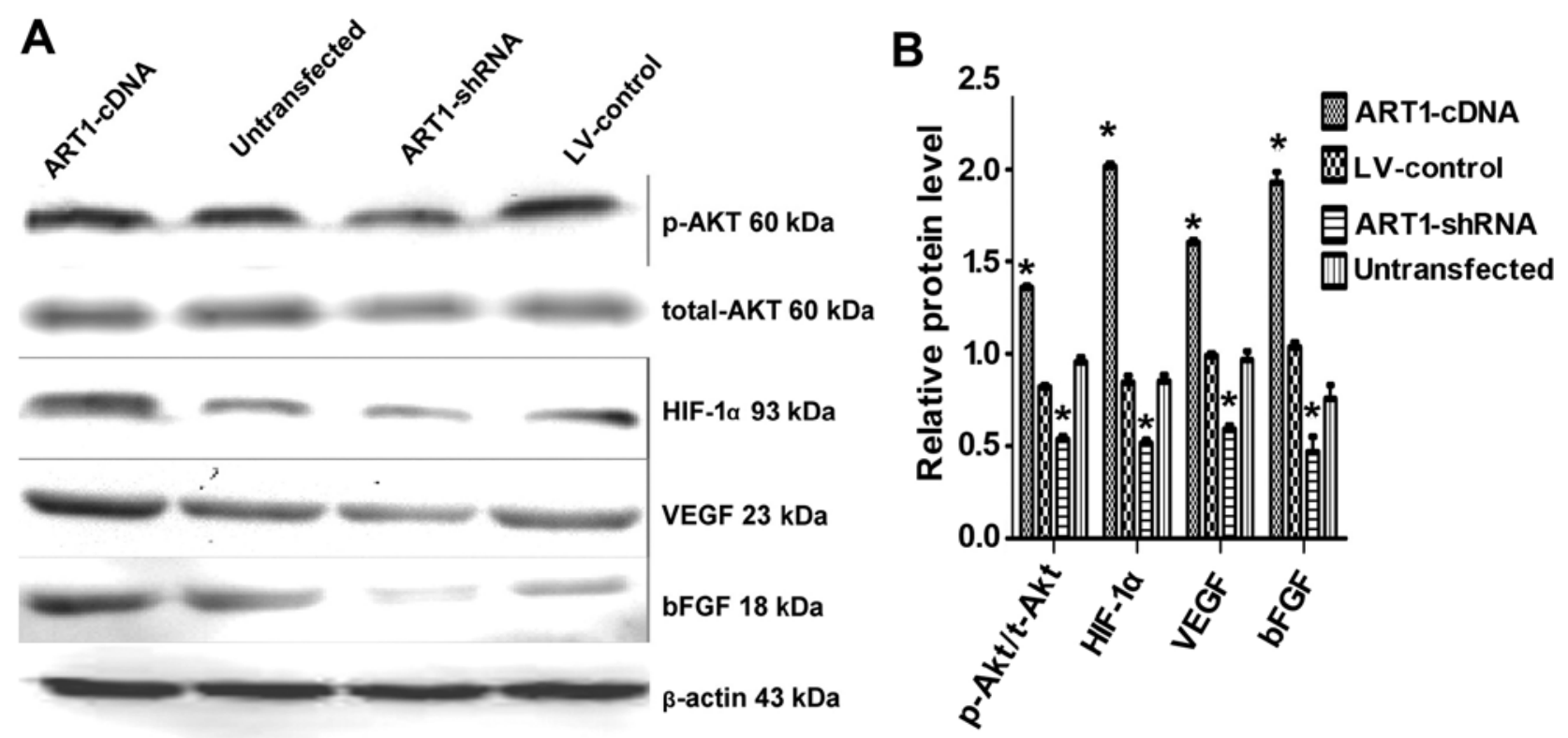

Figure 6. Arginine-specific adenosine diphosphate (ADP)-ribosyltransferase 1 (ART1) affects total-Akt, p-Akt, HIF-1 $\alpha$, VEGF and bFGF expression in LoVo cells (A) The expression of total-Akt, p-Akt, hypoxia-inducible factor 1- $\alpha$ (HIF-1- $\alpha$ ), vascular endothelial growth factor (VEGF) and basic fibroblast growth factor (bFGF) in ART1-mediated LoVo cells. (B) Comparison of Akt, p-Akt, HIF-1 $\alpha$, VEGF and bFGF expression in ART1-cDNA-transfected LoVo cells with that of ART1shRNA-transfected LoVo cells, LV-control-transfected LoVo cells and untransfected LoVo cells. " $\mathrm{P}<0.05$ vs. untransfected LoVo cells; $\mathrm{n}=3$ experiments.

Effects of ART1 on the expression of $t$-Akt, $p$-Akt, HIF-l $\alpha$, $V E G F$ and $b F G F$. In comparison with the control and also the untransfected groups, we noted that p-Akt, HIF-1 $\alpha$, VEGF and bFGF expression were increased in the ART1-cDNA-transfected group, while expression was decreased in the ART1-shRNAtransfected group $(\mathrm{P}<0.05)$. The expression of $\mathrm{t}-\mathrm{Akt}$ did not differ significantly between the experimental groups and the control groups $(\mathrm{P}>0.05)$. Furthermore, $\mathrm{p}-\mathrm{Akt}, \mathrm{HIF}-1 \alpha$, VEGF and bFGF expression did not differ significantly between the untransfected LoVo cells and LV-control-transfected LoVo cells (Fig. 6).

\section{Discussion}

ART1, a representative ecto-ART family protein, catalyzes ADP-ribosylation, which transfers an ADP-ribose from NAD ${ }^{+}$ to an amino acid residue, and thus plays an important role in the protein modification mechanism. Pioneering research has found that certain growth factors are directly regulated by ART1, and ART1 modulates the binding of PDGF-BB to its receptor and regulates its signal transduction capabilities (12). Our earlier studies have demonstrated that ART1 is related to apoptosis, proliferation and migration of mouse CT26 cells (28-30), suggesting that ART1 is involved in tumor growth, invasion and metastasis. It is widely known that angiogenesis plays an important role in malignant tumor occurrence, development, invasion and metastasis, but as yet there have been no studies regarding the role of ART1 in angiogenesis during CRC.

We found that ART1 was highly expressed in a human $\mathrm{CRC}$ and was positively correlated with the expression of antigenic factors (19). In order to further confirm the association between ART1 and angiogenesis, in the present study, ART1 was stably expressed at a high level in the LoVo cell line and silenced using lentiviral vectors. Additionally, LoVo cells were co-cultured with HUVECs to determine the effects of high expression of ART1 or ART1 silencing on HUVEC proliferation, migration and angiogenesis. Our data demonstrated that in LoVo cells with high ART1 expression, HUVEC proliferation, migration and angiogenesis was increased relative to the control. By contrast, HUVEC proliferation, migration and angiogenesis were significantly reduced following co-culture with ART1-silenced LoVo cells. These data suggest that ART1 promotes the proliferation and migration of vascular endothelial cells, thereby inducing tumor angiogenesis.

VEGF is a crucial mitogen for vascular endothelial cells in vitro and in vivo, and plays an important role in inducing endothelial cell proliferation and angiogenesis. VEGF has been shown to correlate with angiogenesis in a number of human cancers, as evidenced by MVD $(31,32)$. bFGF is the strongest growth factor to induce cell generation, and increases the expression of VEGF in endothelial cells while directly contributing to the proliferation of vascular endothelial cells, exerting a synergistic effect with VEGF (33). VEGF/bFGF-induced angiogenesis is a crucial step in tumor progression and metastasis. The data in our present study showed that compared with the control lentivirus-transfected group and untransfected group, VEGF and bFGF expression was upregulated in the group transfected with ART1-cDNA, while expression was downregulated in silenced ART1 LoVo cells. MVD was significantly higher in tumor tissues of the mice which had been infected with CT26 cells transfected with ART1-cDNA, whereas angiogenesis was inhibited when ART1 was silenced in the intrasplenic implantation model. These results suggest that ART1 promotes the expression of VEGF and bFGF, but its mechanism is not yet clear.

Our previous research has shown that the ART1-specific inhibitor MIBG inhibits the expression of the integrin $\alpha 7 \beta 1$, 
focal adhesion kinase (FAK) and PI3K, thereby inhibiting HepG2 cell growth and invasion (34). Another study has shown that ARTs directly regulate phosphodiesterase in the retina (35), suggesting that ARTs affects protein functions by directly regulating ADP-ribosylation, thus modulating signal transduction. It has previously been deonstrated that multiple signaling networks are involved in the regulation of tumor angiogenesis, and the PI3K/Akt pathway has been shown to be of significant importance (36).

Akt is a major downstream effector molecule of PI3K (37), and increased $\mathrm{Akt}^{\mathrm{Thr} 308}$ phosphorylation implies the augmentation of the PI3K pathway (38); it can directly phosphorylate various transcription factors such as NF- $\kappa \mathrm{B}$ and mTOR and is involved in the regulation of various biological activities (39). PI3K activation induces Akt phosphorylation, thus promoting GSK-3 phosphorylation, inhibiting HIF-1 $\alpha$ degradation (40), activating the downstream gene mTOR and promoting HIF-1 $\alpha$ gene transcription (41). HIF-1 $\alpha$ has been revealed to play a central role in regulating the metabolic switch in cancer (42). Overexpression of HIF-1 $\alpha$ is related to increased mortality in patients with various types of tumor $(43,44) \mathrm{HIF}-1 \alpha$ is activated by PI3K-Akt-mTOR pathways and hyper-activity of RAS/MAPK (45). It is also activated by oncogenic mutation of PTEN and VHL $(46,47)$. Activated HIF- $1 \alpha$ binds to the hypoxia response element (HREs), promoting the transcription of a variety of pro-angiogenic genes in the cell and cellular matrix and upregulating VEGF, bFGF expression and that of other angiogenesis-related factors in tumor tissues (48). HIF-1 $\alpha$ regulates the expression of VEGF in cancer cells, is activated by growth factors, and induces formation of the proangiogenic microenvironment in gastric cancer (45). The CD31-positive vessels and tumor growth both decreased in a subcutaneous implantation model when HIF-1 $\alpha$ activation was blocked in a gastric cancer cell line (49). These results suggest that suppression of Thr PI3K/Akt pathway leads to decreased expression of HIF-1 $\alpha$ and inhibits tumor angiogenesis.

Based on these studies, in the present study we detected the expression of t-Akt, p-Akt and HIF-1 $\alpha$, and the data showed that compared with the control groups, p-Akt, and HIF-1 $\alpha$ expression was upregulated in LoVo cells transfected with ART1-cDNA, whereas expression was downregulated in LoVo cells transfected with ART1-shRNA. We speculate that ART1 regulates HIF-1 $\alpha$ expression through the PI3K/Akt pathway. Similar to the effects of ART1 silencing, the PI3K /Akt pathway inhibitor LY294002 inhibited Akt phosphorylation but failed to bring about significant changes in ART1 expression (28), and this confirms that the Akt pathway is located downstream of ART1. These data suggest that ART1 promotes Akt phosphorylation and subsequent HIF-1 $\alpha$ expression and activation, increases VEGF and bFGF expression of cancer cells, and eventually leads to an increase in tumor angiogenesis in tumor tissue.

In brief, these data suggest that the mechanism of ART1 in tumor angiogenesis is quite complex and is involved in multiple signaling pathways. The data from the present study demonstrate that ART1 upregulates HIF-1 $\alpha$ through the PI3K/ Akt signaling pathway to promote the expression of angiogenic factors such as VEGF and bFGF and induce angiogenesis in cancer tissue. Furthermore, its exact mechanisms require further in-depth study.

\section{Acknowledgements}

This study was supported by the Ministry of Education Specialized Research Fund for the Doctoral Program of Higher Education (Grant no. 20105503110009), the Science and Technology Project of Chongqing Municipal Education Commission (Grant no. KJ110322) and the National Nature Science Foundation of China (NSFC: 30870946). We would like to thank Tang Yi and Xiao Ming for preparing the CT26 murine CRC cells, and we also thank Kuang Jing, and Song Guang-Lin, postgraduate students of Chongqing Medical and Science University, for their assistance in this study.

\section{References}

1. Bergers G, Brekken R, McMahon G, Vu TH, Itoh T, Tamaki K, Tanzawa K, Thorpe P, Itohara S, Werb Z and Hanahan D: Matrix metalloproteinase-9 triggers the angiogenic switch during carcinogenesis. Nat Cell Biol 2: 737-744, 2000.

2. Seman M, Adriouch S, Haag F and Koch-Nolte F: Ecto-ADPribosyltransferases (ARTs): Emerging actors in cell communication and signaling. Curr Med Chem 11: 857-872, 2004.

3. Braren R, Glowacki G, Nissen M, Haag F and Koch-Nolte F: Molecular characterization and expression of the gene for mouse $\mathrm{NAD}^{+}$: arginine ecto-mono(ADP-ribosyl)transferase, Art1. Biochem J 336: 561-568, 1998.

4. Corda D and Di Girolamo M: Functional aspects of protein mono-ADP-ribosylation. EMBO J 22: 1953-1958, 2003.

5. Okazaki IJ, Zolkiewska A, Nightingale MS and Moss J: Immunological and structural conservation of mammalian skeletal muscle glycosylphosphatidylinositol-linked ADP-ribosyltransferases. Biochemistry 33: 12828-12836, 1994.

6. Kefalas P, Allport JR, Donnelly LE, Rendell NB, Murray S, Taylor GW, Lo G, Yadollahi-Farsani M and MacDermot J: Arginine-specific mono(ADP-ribosyl)transferase activity in human neutrophil polymorphs. A possible link with the assembly of filamentous actin and chemotaxis. Adv Exp Med Biol 419: 241-244, 1997.

7. Balducci E, Horiba K, Usuki J, Park M, Ferrans VJ and Moss J: Selective expression of RT6 superfamily in human bronchial epithelial cells. Am J Respir Cell Mol Biol 21: 337-346, 1999.

8. Zolkiewska A and Moss J: Integrin alpha 7 as substrate for a glycosylphosphatidylinositol-anchored ADP-ribosyltransferase on the surface of skeletal muscle cells. J Biol Chem 268: 25273-25276, 1993.

9. Nemoto E, Yu Y and Dennert G: Cell surface ADP-ribosyltransferase regulates lymphocyte function-associated molecule-1 (LFA-1) function in T cells. J Immunol 157: 3341-3349, 1996.

10. Wang J, Nemoto E and Dennert G: Regulation of CTL by ecto-nictinamide adenine dinucleotide (NAD) involves ADP-ribosylation of a p56lck-associated protein. J Immunol 156: 2819-2827, 1996.

11. Laing S, Unger M, Koch-Nolte F and Haag F: ADP-ribosylation of arginine. Amino Acids 41: 257-269, 2011.

12. Saxty BA, Yadollahi-Farsani M, Upton PD, Johnstone SR and MacDermot J: Inactivation of platelet-derived growth factor-BB following modification by ADP-ribosyltransferase. Br J Pharmacol 133: 1219-1226, 2001.

13. Kato J, Zhu J, Liu C and Moss J: Enhanced sensitivity to cholera toxin in ADP-ribosylarginine hydrolase-deficient mice. Mol Cell Biol 27: 5534-5543, 2007.

14. Del Vecchio $M$ and Balducci E: Mono ADP-ribosylation inhibitors prevent inflammatory cytokine release in alveolar epithelial cells. Mol Cell Biochem 310: 77-83, 2008.

15. Stevens LA, Levine RL, Gochuico BR and Moss J: ADP-ribosylation of human defensin HNP-1 results in the replacement of the modified arginine with the noncoded amino acid ornithine. Proc Natl Acad Sci USA 106: 19796-19800, 2009.

16. Liu ZX, Yu Y and Dennert G: A cell surface ADP-ribosyltransferase modulates $\mathrm{T}$ cell receptor association and signaling. J Biol Chem 274: 17399-17401, 1999.

17. Akai T, Nabeya Y, Yahiro K, Morinaga N, Mitsuhashi K, Inoue M, Sakamoto A, Ochiai T and Noda M: Helicobacter pylori induces mono-(adenosine 5'-diphosphate)-ribosylation in human gastric adenocarcinoma. Int J Oncol 29: 965-972, 2006. 
18. Balducci E, Micossi LG, Soldaini E and Rappuoli R: Expression and selective up-regulation of toxin-related mono ADP-ribosyltransferases by pathogen-associated molecular patterns in alveolar epithelial cells. FEBS Lett 581: 4199-4204, 2007.

19. Yang L, Wang YL, Sheng YT, Xiong W, Xu JX and Tang Y and Li X: The correlation of ART1 expression with angiogenesis in colorectal carcinoma and its relationship with VEGF and integrin $\alpha \mathrm{V} \beta 3$ expressions. J Basic Clin Med 32: 1065-1069, 2012.

20. Fang J, Ding M, Yang L, Liu LZ and Jiang BH: PI3K/PTEN/AKT signaling regulates prostate tumor angiogenesis. Cell Signal 19: 2487-2497, 2007.

21. Gray MJ, Zhang J, Ellis LM, Semenza GL, Evans DB Watowich SS and Gallick GE: HIF-1alpha, STAT3, CBP/p300 and Ref-1/APE are components of a transcriptional complex that regulates Src-dependent hypoxia-induced expression of VEGF in pancreatic and prostate carcinomas. Oncongene 24: 3110-3120, 2005.

22. Tang Y, Wang Y-L, Yang L, Xu J-X, Xiong W, Xiao M and Li M: Inhibition of arginine ADP-ribosyltransferase 1 reduces the expression of poly(ADP-ribose) polymerase-1 in colon carcinoma. Int J Mol Med 32: 130-136, 2013.

23. Yang LP, Cheng P, Peng XC, Shi HS, He WH, Cui FY, Luo ST Wei YQ and Yang L: Anti-tumor effect of adenovirus-mediated gene transfer of pigment epithelium-derived factor on mouse B16-F10 melanoma. J Exp Clin Cancer Res 28: 75-78, 2009.

24. Huang C, Yuan X, Li Z, Tian Z, Zhan X, Zhang J and Li X VE-statin/Egfl7 siRNA inhibits angiogenesis in malignant glioma in vitro. Int J Clin Exp Pathol 7: 1077-1084, 2014.

25. Rajesh M, Mukhopadhyay P, Godlewski G, Bátkai S, Haskó G, Liaudet L and Pacher P: Poly(ADP-ribose)polymerase inhibition decreases angiogenesis. Biochem Biophys Res Commun 350: 1056-1062, 2006.

26. Neo JH, Malcontenti-Wilson C, Muralidharan V and Christophi C: Effect of ACE inhibitors and angiotensin II receptor antagonists in a mouse model of colorectal cancer liver metastases. J Gastroenterol Hepatol 22: 577-584, 2007.

27. Weidner N, Semple JP, Welch WR and Folkman J: Tumor angiogenesis and metastasis - correlation in invasive breast carcinoma. N Engl J Med 324: 1-8, 1991

28. Xiao M, Tang Y, Wang YL, Yang L and Li X, Kuang J and Song GL: ART1 silencing enhances apoptosis of mouse CT26 cells via the PI3K/Akt/NF-кB pathway. Cell Physiol Biochem 32: 1587-1599, 2013.

29. Xu JX, Wang YL, Tang Y and Xiong W: RNA interference of ART1 on colon cancer cell proliferation in mice and its mechanism. Tumor 32: 949-954, 2012.

30. Wei X, Tang Y, Wang Y and Xu J-X: Effects of ART1 gene silencing on the ability of CT26 cellular matrix adhesion and migration. Fudan Univ J Med Sci 40: 328-334, 2013.

31. Imura $S$, Miyake $H$, Izumi K, Tashiro $S$ and Uehara $H$ : Correlation of vascular endothelial cell proliferation with microvessel density and expression of vascular endothelial growth factor and basic fibroblast growth factor in hepatocellular carcinoma. J Med Invest 51: 202-209, 2004

32. Raspollini MR, Castiglione F, Garbini F, Villanucci A, Amunni G, Baroni G, Boddi V and Taddei GL: Correlation of epidermal growth factor receptor expression with tumor microdensity vessels and with vascular endothelial growth factor expression in ovarian carcinoma. Int J Surg Pathol 13: 135-142, 2005.
33. Eriksson K, Magnusson P, Dixelius J, Claesson-Welsh L and Cross MJ: Angiostatin and endostatin inhibit endothelial cell migration in response to FGF and VEGF without interfering with specific intracellular signal transduction pathways. FEBS Lett 536: 19224, 2003

34. Su Y, Guan XQ, Liu FQ and Wang YL: The effects of MIBG on the invasive properties of HepG2 hepatocellular carcinoma cells. Int J Mol Med 34: 842-848, 2014.

35. Bondarenko VA and Yamazaki A: Characterization of argininespecific mono-ADP ribosyltransferase isolated from frog retina and its function in signal transduction. Invest Ophthalmol Vis Sci 50: 5440-A391, 2009.

36. Kerbel RS: Tumor angiogenesis. N Engl J Med 358: 2039-2049, 2008.

37. McCubrey JA, Steelman LS, Chappell WH, Abrams SL, Montalto G, Cervello M, Nicoletti F, Fagone P, Malaponte G, Mazzarino MC, et al: Mutations and deregulation of Ras/Raf/ MEK/ERK and PI3K/PTEN/Akt/mTOR cascades which alter therapy response. Oncotarget 3: 954-987, 2012.

38. Hart JR and Vogt PK: Phosphorylation of AKT: a mutational analysis. Oncotarget 2: 467-476, 2011.

39. Sheng S, Qiao M and Pardee AB: Metastasis and AKT activation. J Cell Physiol 218: 451-454, 2009.

40. Hawkins PT, Anderson KE, Davidson K and Stephens LR: Signalling through class I PI3Ks in mammalian cells. Biochem Soc Trans 34: 647-662, 2006.

41. Agani F and Jiang BH: Oxygen-independent regulation of HIF-1: novel involvement of PI3K/AKT/mTOR pathway in cancer. Curr Cancer Drug Targets 13: 245-251, 2013.

42. Semenza GL: HIF-1: Upstream and downstream of cancer metabolism. Curr Opin Genet Dev 20: 51-56, 2010.

43. Lu X and Kang Y: Hypoxia and hypoxia-inducible factors: master regulators of metastasis. Clin Cancer Res 16: 5928-5935, 2010.

44. Rohwer $\mathrm{N}$ and Cramer T: HIFs as central regulators of gastric cancer pathogenesis. Cancer Biol Ther 10: 383-385, 2010.

45. Kitajima Y and Miyazaki K: The critical impact of HIF-1 $\alpha$ on gastric cancer biology. Cancers (Basel) 5: 15-26, 2013.

46. Lee BL, Kim WH, Jung J, Cho SJ, Park JW, Kim J, Chung HY, Chang MS and Nam SY: A hypoxia-independent up-regulation of hypoxia-inducible factor- 1 by AKT contributes to angiogenesis in human gastric cancer. Carcinogenesis 29: 44-51, 2008.

47. Chun SY, Johnson C, Washburn JG, Cruz-Correa MR, Dang DT and Dang LH: Oncogenic KRAS modulates mitochondrial metabolism in human colon cancer cells by inducing HIF-1 $\alpha$ and HIF-2 $\alpha$ target genes. Mol Cancer 9: 293, 2010.

48. Pugh CW and Ratcliffe PJ: Regulation of angiogenesis by hypoxia-inducible factor-1: role of the HIF system. Nat Med 9: 677-684, 2003

49. Lang SA, Gaumann A, Koehl GE, Seidel U, Bataille F, Klein D, Ellis LM, Bolder U, Hofstaedter F, Schlitt HJ, et al: Mammalian target of rapamycin is activated in human gastric cancer and serves as a target for therapy in an experimental model. Int J Cancer 120: 1803-1810, 2007. 